\title{
Performance Characteristics of Malaria Rapid Diagnostic Test and Its Utilization in Management of Febrile Patients in Korogwe District (Tanga) Tanzania
}

\author{
Kachua Ndewa $^{1,2, *}$, Charles M. Kihamia ${ }^{1}$, Marycelina Mubi ${ }^{1}$, George Semango ${ }^{3}$ \\ ${ }^{1}$ Muhimbili University of Health and Allied Sciences, Tanzania \\ ${ }^{2}$ Korogwe District Hospital, Tanzania \\ ${ }^{3}$ Kilimanjaro Christian Medical Centre, Tanzania
}

Copyright $\mathrm{O} 2016$ by authors, all rights reserved. Authors agree that this article remains permanently open access under the terms of the Creative Commons Attribution License 4.0 International License

\begin{abstract}
Background: Malaria diagnosis continues to be the foremost among the challenge in malaria control strategies in Sub-Saharan countries including Tanzania. The World Health Organization changed malaria treatment Guidelines in 2010 to restrict the use of antimalarial drugs to parasitologically confirmed malaria cases in response to the overuse of antimalarial drugs. Malaria rapid diagnostic tests (mRDTs) are presented as a mean to implement the new guidelines for malaria parasitologically confirmed cases prior to initiating antimalarial drug therapy for mRDT positive patients. Methods: A hospital-based cross-sectional study among patients with fever and malaria-like symptoms was done between May and June, 2014 in Korogwe district hospital in a convenient manner. Data were cleaned and analysed in Epi-Info version 3.5.4 computer software, Chi-square test was used to compare proportions between two groups with P-value set at 0.05 . Results: Results revealed that sensitivity, specificity, positive predictive value and negative predictive value of mRDT at $95 \% \mathrm{CI}$ was $97.6 \%, 97.4 \%, 91.0 \%$ and $99.3 \%$ respectively. All patients with positive mRDT were treated with antimalarial drugs, while patients with negative $\mathrm{mRDT}$ results were treated with either antimalarial $(\mathrm{P}$-value $=0.001)$ or antibiotics $(\mathrm{P}$-value $=$ $0.005)$ at $95 \%$ CI. The results of patients with negative mRDT results were less likely to be further investigated at 95\% CI (P-value $=1.000)$. Conclusion: The mRDT had good sensitivity and specificity if compared with blood slide microscopy. Clinicians adhered to the 2010 World Health Organization guidelines adopted by the National Malaria Control Programme for patients with positive mRDT result; however, they did not adhere to malaria diagnosis and management guidelines in case of negative mRDT results.
\end{abstract}

Keywords Malaria Diagnosis, mRDT Sensitivity and Specificity, Overuse of Antimalarial Drugs, Clinicians Adherence to 2010 World Health Organization Guidelines

\section{Background}

Malaria is a major global public health dilemma that exerts a high mortality and morbidity in many countries, particularly in resource-poor countries in Sub-Saharan Africa, South East Asia and Latin America, and probably contributes to keep these countries in poverty [1]. The World Health Organization estimates that 3.4 billion people globally are at risk of malaria and 0.6 million deaths occur due to malaria (90\% in Africa) most being children and pregnant women [2]. Diagnosis of malaria is still a challenge mainly based on increasing presumptive malaria treatment, and non-adherence to malaria parasitological confirmed negative results. Malaria over diagnosis, still practiced around the world, results in unnecessary use of antimalarial drugs, exaggeration of the burden of the disease, delay of appropriate treatment and sometimes death due to non-malaria causes of fever [3].

A clinical diagnosis of malaria is a traditional method of management of patients with fever and most practiced in developing world, Tanzania being one of them. This method is least expensive and most widely practiced in peripheral setting. Clinical diagnosis is based on the patient's signs, symptoms and on findings of physical examination. This method is fast but it lacks precision due to other diseases presenting with the same signs and symptoms like malaria such as common viral or bacterial infections and other febrile illnesses [4-7]. When managing febrile patients, it is impossible to know if the condition is due to malaria or other disease on the basis of clinical presentation hence an accurate diagnosis is essential. Laboratory diagnosis is an essential component of malaria control and elimination strategy that promises accurate diagnosis and treatment with effective medication within 24 hours of onset of illness if patients use effective medication. When managing febrile patients, it is impossible to know if the condition is due to malaria or other disease on the basis of clinical presentation 
hence an accurate diagnosis is essential. As the incidence of malaria has diminished globally, a better understanding of non-malarial fever is important for effective management of patient's illness and rational use of antimalarial drugs. Globally malaria diagnosis by microscope is gold standard diagnostic method with high sensitivity and specificity provided that the quality of malaria microscopy is good. When good materials and skilled technicians are available, malaria microscopy is an excellent method to diagnose malaria as it can detect malaria parasites as few as 20 malaria parasites $/ \mu$ l. Malaria Rapid Diagnostic tests (mRDTs) are devices that assist in the diagnosis of malaria by providing evidence of the presence of malaria parasites in human blood within a short time (15 to 20 minutes). Malaria rapid diagnostic tests are easy and quick to perform as they can be performed easily by any health professional that has been given a short training. This method is cost-effective especially when Artemether-Lumefantrine (ALu) is used as first line treatment for malaria [8]. Malaria case management could be improved by using mRDTs as this method can be used even in areas where microscopy diagnosis is impractical [9]. Therefore mRDT is a reliable test for diagnosis and patient management in areas where blood slide microscopy is impractical [10-12].

\section{Materials and Methods}

\section{Study Design}

This was a hospital-based cross-sectional study of all patients with fever and malaria-like symptoms. The study was conducted between May and June 2014, to assess performance characteristics of malaria rapid diagnostic test and its utilization in the management of febrile patients.

\section{Study Population}

All patients with fever and malaria-like symptoms, attending health care and treatment in outpatient clinic at Korogwe district hospital. In this study fever is defined as body temperature above $37.5{ }^{\circ} \mathrm{C}$ while malaria-like symptoms are signs and symptoms of malaria like headache, muscles aches, tiredness, nausea, vomit and diarrhea associated with fever.

\section{Sampling Technique}

The sample was obtained on convenience basis and the patients available at the time of data collection were selected. All outpatients reporting complaints of fever and malarialike symptoms to the reception were given the opportunity to be included in the study through convenience sampling. Fever was defined as body temperature above $37.5^{\circ} \mathrm{C}$ or raised body temperature within the past 24 hours. The selected individuals were given laboratory identification number and enrolled for the test after they signed the informed consent form.

\section{Data Collection Procedures}

A structured questionnaire was administered to obtain the demographic information after patients were seen by the clinician and the clinical diagnosis of malaria was made. In the laboratory, blood sample for mRDT, thin and thick smears for Giemsa staining technique were taken and labeling was done on the frosted part of the slide using a pencil. Labeling included the initials of patient name, identification number, date, month and year in which the specimen was collected. The principal investigator performed all the malaria rapid diagnostic test and the results were used to treat patients. Both field laboratory technicians and the third slide reader at Muhimbili University of Health and Allied Sciences were blinded to mRDT results. Stained slides were handed over to the field technician with specimen tracking form where he/she entered his/her results daily. After the clinician attended to the patients, the questionnaire form was filled by a nurse in order to record the patient's management decision from the prescriber (Exit interview).

\section{Ethical Considerations}

Ethical approval for this study was obtained from Muhimbili University of Health and Allied Sciences (MUHAS) through the MUHAS IRB committee. Permission for dissertation field work was obtained from the Korogwe district Executive Director and the Korogwe District Medical Officer.

\section{Diagnostic Tool}

The SD BIOLINE Malaria Ag P.f/Pan test cassette contains a membrane strip, which is precoated with mouse monoclonal antibodies specific to HRP-II of P. falciparum on test line $P f$ region and with mouse monoclonal antibodies specific to lactate dehydrogenase of Plasmodium species (P.falciparum, $P$. vivax, $P$. malariae, $P$. ovale) on test line Pan Region respectively. The mixture of mouse monoclonal antibodies specific to HRP-II of $P . f$ and mouse monoclonal antibodies specific to pLDH of Pan. They move along with membrane chromatographically to the test region $(P f$ and Pan) and form a visible line as the antibody-antigen-antibody gold particle complex formed. All the test lines and control line in the result window are not visible before applying any sample. The control line is used for procedural control and should always appear if the test procedure is performed correctly.

\section{Operative Protocol}

Sample was obtained on convenience-basis from the patients that happened to be available at the time of data collection were selected. The mRDT test result was reported directly by the field laboratory technician. The blood smears were examined for 7- 10 minutes per slide by a qualified laboratory technician in the field as the first reader, followed by the second reader at Korogwe district hospital. For positive smears, the number of parasites was counted in reference to 200 white blood cells (WBCs). The third reading was done by a competent microscopist who was blinded to 
previous results in Dar-es-salaam at Muhimbili University, Parasitology and Entomology department.

\section{Data Processing and Analysis}

Data were entered, cleaned and analysed in Epi-Info version 3.5.4 computer software and presented in form of frequency tables, means and standard deviations. Frequencies and cross tabulation was produced to obtain odd ratio, $P$-value and Chi- square tests, for each of the independent variables at $95 \%$ confidence interval. A Chi-square test, $P$-value, correlation coefficient and kappa value were done in comparing degree of inter-observer agreement between reader one, two and three blood slides microscopy. Statistical significance for statistical tests was achieved when $P$-value is $\leq 0.05$

\section{Results}

\section{Demographic Characteristics}

A total of 2,100 people aged one month to 95 years attended the outpatient department (OPD) at Korogwe district hospital. Out of these, 385 patients were recruited and enrolled in the study based either on malaria-like symptoms or raised body temperature on arrival (body temperature $\geq 37.5$ ).

Table 1 shows that most of participants 245 were female $(63.6 \%)$, while $140(36.4 \%)$ were male, most of participants (79) were in age group of $0-5$ years $(20.5 \%)$ and 40 years $(10.4 \%)$. were in the age group $42-50$ years, as least group.

Table 1. Demographic characteristics of the study population $(\mathrm{N}=385)$

\begin{tabular}{|c|c|c|}
\hline Variable & Number & Percentage (\%) \\
\hline Sex & & \\
\hline Male & 140 & 36.4 \\
\hline Female & 245 & 63.6 \\
\hline Age group (years) & & \\
\hline$<5$ & 79 & 20.5 \\
\hline $5-14$ & 58 & 15.1 \\
\hline $15+$ & 248 & 64.4 \\
\hline
\end{tabular}

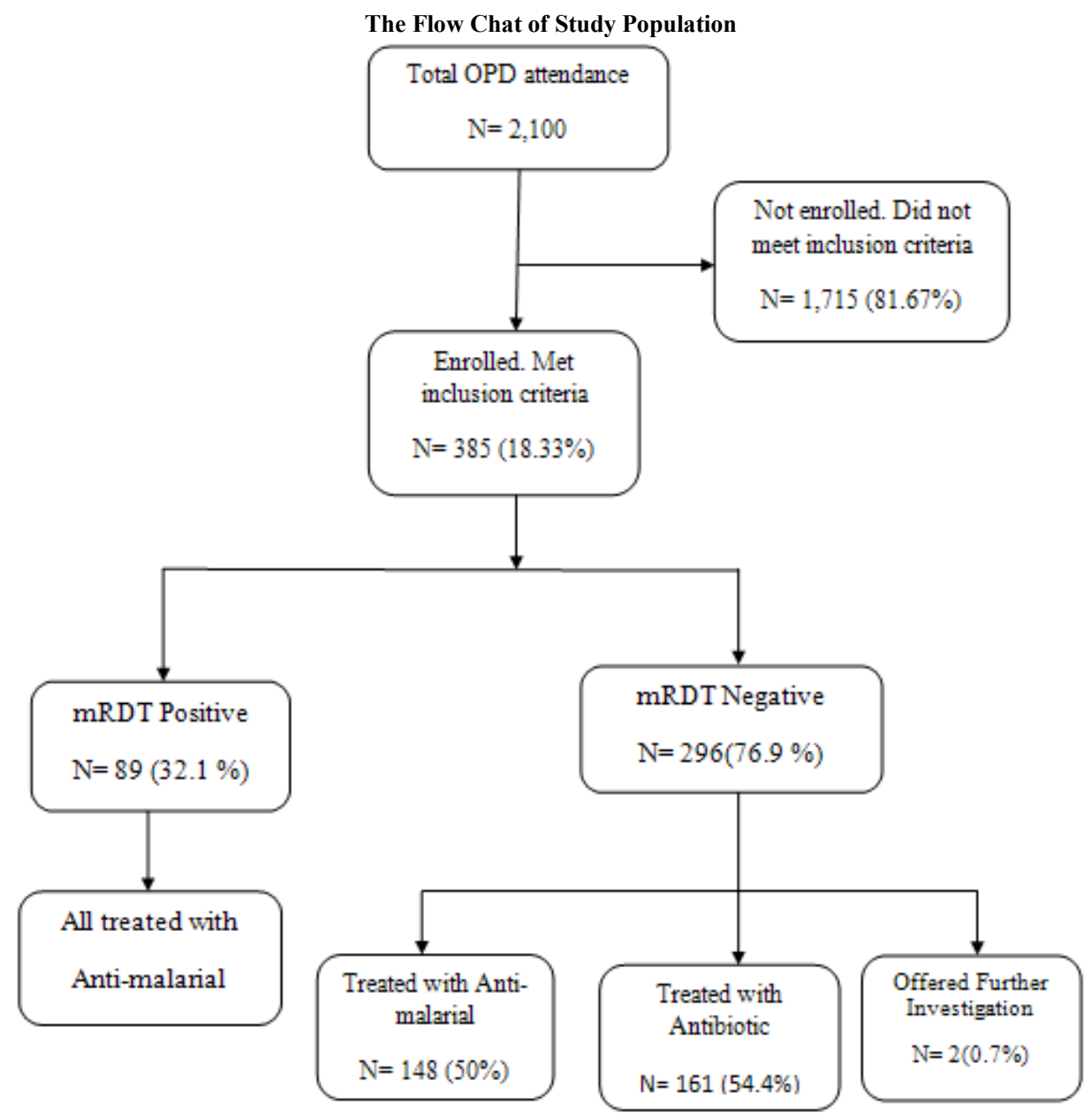

Figure 1. The flow chart for the study population 
Table 2. Hospital based malaria prevalence

\begin{tabular}{|c|c|c|c|c|}
\hline $\begin{array}{c}\text { Age group } \\
\text { (years) }\end{array}$ & Number & Positive & Negative & $\begin{array}{c}\text { Prevalence } \\
(\%)\end{array}$ \\
\hline$<5$ & 79 & 25 & 54 & 31.6 \\
\hline $5-14$ & 58 & 25 & 33 & 43.1 \\
\hline $15+$ & 248 & 39 & 209 & 15.7 \\
\hline Total & 385 & 89 & 296 & 23.1 \\
\hline
\end{tabular}

Table 2 shows that overall hospital malaria prevalence at $95 \%$ CI was $(23.1 \%$ and $21.6 \%)$ by mRDT and BS respectively.

Table 3. Comparison of Giemsa and mRDT results

\begin{tabular}{|c|c|c|c|c|c|c|}
\hline & \multicolumn{3}{|c|}{ Blood slide reader 3} & \multirow[t]{2}{*}{ OD } & \multirow[t]{2}{*}{$x^{2}$} & \multirow{2}{*}{$\begin{array}{c}\mathrm{p}- \\
\text { value }\end{array}$} \\
\hline $\begin{array}{l}\text { mRDT } \\
\text { results }\end{array}$ & Positive & Negative & Total & & & \\
\hline Positive & 81 & 8 & $\begin{array}{c}89 \\
(23.1 \%)\end{array}$ & & & \\
\hline & & & & 1.488 & 3.302 & 0.001 \\
\hline Negative & 2 & 294 & $\begin{array}{c}296 \\
(76.9 \%)\end{array}$ & & & \\
\hline Total & $\begin{array}{c}83 \\
(21.6 \%)\end{array}$ & $\begin{array}{c}302 \\
(78.4 \%)\end{array}$ & $\begin{array}{c}385 \\
(100 \%)\end{array}$ & & & \\
\hline
\end{tabular}

Table 3, shows that the mRDT sensitivity, specificity, positive predictive value and negative predictive value at 95\% CI were $91.0 \%, 99.3 \%, 97.6 \%$ and $97.4 \%$ respectively.

Table 4, shows that slides Reader one had 1 discordant results compared to Reader three as reference reader at $95 \%$ CI; slide Reader two had 4 discordant results compared to Reader three as reference reader at 95\% CI.

\section{Kappa Value}

The degree of agreement between Reader one smear microscopy and Reader three gave kappa value of 0.992 The degree of agreement between Reader two smear microscopy and Reader three gave kappa value of 0.969 (Perfect agreement range, 0.81-1.00). Meaning that there were no significant discordant between Reader one and Reader two in the field compared with Reader three at Parasitology and Entomology MUHAS as reference laboratory.

Table 5, shows that $89(100 \%)$ of positive mRDT prescribed antimalarial at $95 \% \mathrm{CI}\left(X^{2}=72.289, P\right.$-value $=$ 0.000 and $\mathrm{OD}=2.00)$. Out of $296 \mathrm{mRDT}$ negative patients, $148(50 \%)$ prescribed antimalarial, while $148(50 \%)$ prescribed antibiotics and $2(0.7 \%)$ offered further investigation.

Table 4. Comparison between reader 1, 2 and 3 blood slides microscopy

\begin{tabular}{|c|c|c|c|c|c|c|c|}
\hline \multicolumn{8}{|c|}{ Slide Reader 3} \\
\hline & & Positive & Negative & Total & $x^{2}$ & $P$-value & $\mathrm{r}$ \\
\hline & Positive & 82 & 0 & 82 & & & \\
\hline \multirow[t]{2}{*}{$\begin{array}{c}\text { Slides reader } \\
1 \\
\end{array}$} & & & & & 3.791 & 0.001 & 0.999 \\
\hline & Negative & 1 & 302 & 303 & & & \\
\hline \multirow[t]{2}{*}{ Total } & & 83 & 302 & 385 & & & \\
\hline & Positive & 79 & 0 & 79 & & & \\
\hline \multirow[t]{2}{*}{$\begin{array}{c}\text { Slides reader } \\
2 \\
\end{array}$} & & & & & 3.617 & 0.001 & 0.969 \\
\hline & Negative & 4 & 302 & 306 & & & \\
\hline Total & & 83 & 302 & 385 & & & \\
\hline
\end{tabular}

Table 5. Association between mRDT results with further patients management decisions

\begin{tabular}{|c|c|c|c|c|c|}
\hline & \multicolumn{2}{|c|}{ Antimalarial } & \multicolumn{2}{c|}{ Antibiotics } & \multicolumn{2}{c|}{ Further investigation } \\
\hline mRDT results & Yes & No & Yes & No & Yes \\
\hline Positive (89) & $89(100 \%)$ & 0 & 20 & 69 & 0 \\
\hline Negative (296) & $148(50 \%)$ & $148(50 \%)$ & $161(54.4 \%)$ & $135(45.6 \%)$ & $2(0.7 \%)$ \\
\hline & \multicolumn{2}{|c|}{$x^{2}=72289$} & $x^{2}=27.987$ & $x^{2}=0.604$ \\
\hline & \multicolumn{2}{|c|}{$P-$-value $=0.000$} & P-value $=0.005$ & P-value $=1.0$ \\
\hline \multicolumn{2}{|c|}{ OD $=2.0$} & OD $=0.243$ & OD $=0.007$ \\
\hline
\end{tabular}


Table 6. Clinical characteristics by mRDT results

\begin{tabular}{|c|c|c|c|c|c|c|}
\hline \multirow[t]{2}{*}{ Variables } & \multicolumn{2}{|c|}{ mRDT Results } & \multirow[b]{2}{*}{ Total } & \multirow[b]{2}{*}{ OD } & \multirow[b]{2}{*}{$x^{2}$} & \multirow[b]{2}{*}{$P$-value } \\
\hline & Positive & Negative & & & & \\
\hline \multicolumn{7}{|l|}{ Body Temperature } \\
\hline $\operatorname{Normal}\left(36.9-37.4^{\circ} \mathrm{C}\right)$ & $35(39.3 \%)$ & $177(59.8 \%)$ & 212 & 0.436 & 11.589 & 0.001 \\
\hline Fever $\left(\geq 37.5^{\circ} \mathrm{C}\right)$ & $54(60.7 \%)$ & $119(40.2 \%)$ & 173 & & & \\
\hline Total & 89 & 296 & 385 & & & \\
\hline \multicolumn{7}{|l|}{ Intermittent fever } \\
\hline Yes & $61(68.5 \%)$ & $141(47.6 \%)$ & 202 & 2.395 & 11.99 & 0.001 \\
\hline No & $28(31.5 \%)$ & $155(52.4 \%)$ & 183 & & & \\
\hline Total & 89 & 296 & 385 & & & \\
\hline \multicolumn{7}{|l|}{ Body malaise } \\
\hline Yes & $89(100 \%)$ & $285(96.3 \%)$ & 374 & 0.762 & 3.405 & 0.75 \\
\hline No & $0(0 \%)$ & $11(3.7 \%)$ & 11 & & & \\
\hline Total & 89 & 296 & 385 & & & \\
\hline \multicolumn{7}{|l|}{ Abdominal discomfort } \\
\hline Yes & $62(69.7 \%)$ & $168(56.8 \%)$ & 230 & 1.75 & 4.739 & 0.36 \\
\hline No & $27(30.3 \%)$ & $128(43.2 \%)$ & 155 & & & \\
\hline Total & 89 & 296 & 385 & & & \\
\hline \multicolumn{7}{|l|}{ Diarrhea } \\
\hline Yes & $27(30.3 \%)$ & $69(23.3 \%)$ & 96 & 1.433 & 1.805 & 0.21 \\
\hline No & $62(69.7 \%)$ & $227(76.7 \%)$ & 289 & & & \\
\hline Total & 89 & 296 & 385 & & & \\
\hline
\end{tabular}

Table 6 above, $54(60.7 \%)$ patients had fever and were $\mathrm{mRDT}$ positive at $95 \% \mathrm{CI}(P$-value $=0.001)$. Patients having intermittent fever and mRDT positive were $61(68.5 \%)$ at $95 \%$ CI $(P$-value $=0.001)$. While body malaise, abdominal discomfort and diarrhea had none association with mRDT positivity at $95 \% \mathrm{CI}$

\section{Discussion}

The study was conducted during the rainy season; malaria positivity was higher with mRDTs than with microscopy. The study reports the overall hospital malaria prevalence of $23.1 \%$ and $21.6 \%$ by mRDT and microscopic blood slide respectively (On table 2). Malaria positivity was higher with mRDT than with microscopy and this could either be due to the strict lower cut-off point for microscopy or higher false positivity of mRDT due to persistent antigen in blood [13]. The study found that sensitivity, specificity, positive predictive value, negative predictive value of mRDT (Ag. $\mathrm{pLDH} / \mathrm{HRP} 2$ ) were $97.6 \%, 97.4 \%, 91.0 \%$ and $99.3 \%$ respectively in comparison with blood slides microscopy (On table 3) [6]. Out of 385 participants screened for malaria by mRDT, $89(23.1 \%)$ were malaria positive and treated according to malaria diagnosis and treatment guidelines. The remaining portion was mRDT negative, even if they presented signs and symptoms of malaria. Fever has significant association with malaria case at $95 \% \mathrm{CI}$, it is the best symptom of malaria on clinical judgment but it is not specific for malaria case. Intermittent fever has a significant association with a malaria case at $95 \% \mathrm{CI}$, is the best symptom to be used in clinical judgment of malaria. Body malaise, abdominal discomfort and diarrhea all at 95\% CI had non-association with malaria case, basing on that, it is better not to use such symptoms in clinical judgment, because they are not specific for malaria case (On table 6). In a similar study done in high transmission areas (Benin), patients have acquired a partial immunity to malaria and when infected they present few or no symptoms. Fever, body malaise and intermittent fever were significantly associated with a malaria infection and almost $90 \%$ of infected patients presented at least one of these symptoms [14]. This study showed that all $89(100 \%)$ positive patients by mRDT were prescribed antimalarial, while $296(100 \%)$ negative patients by mRDT were prescribed either antimalarial $148(50 \%)$ or antibiotic $148(50 \%)$. There is a significant association of positive mRDT with prescription of antimalarial at $95 \% \mathrm{CI}$. Few $20(22.2 \%)$ were prescribed both antimalarial and antibiotics; these are under five years children. For negative mRDT 296 (76.9\%) there were significant associations for prescribed antimalarial or antibiotics at $95 \%$ CI. There was no association for negative mRDT result to offer further investigation at $95 \%$ CI. The World Health Organization guidelines of 2010 adopted by NMCP for diagnosis and 
treatment of malaria were full adherent to positive mRDT patients but not adherence to negative mRDT ones (On table 5) $[5,15-17]$. On the other hand published evidence indicates that in many instances, adherence to mRDT test results is very poor in negative patients compared to positive patients [1]. Other studies showed that antimalarial drugs were prescribed to all patients with positive test results, while in patients with negative results Antibiotics were more likely to be prescribed compared to patients with positive results [18-20]. In a study done in Tanzania (Beyond fever) which included 1005 children with temperature of 38 degrees, a total of $70 \%$ had viral disease, $22 \%$ had bacteria disease and only $10.9 \%$ had parasitic disease [21]. Based on this data high temperature does not confirm malaria case and to offer Anti-malaria to mRDT negative patients is over-use of anti-malaria drugs.

\section{Limitations}

The data were collected one month after heavy rains, might contribute malaria positivity compared to other season.

\section{Conclusions}

In rainy season, hospital-based malaria prevalence is still high and children under five years are the most affected age group. The mRDT (Ag. pLDH/HRP-2) manufactured by Premier Medical Corporation Limited (India) and distributed by Ministry of Health Tanzania through Medical Store Department have good sensitivity and specificity as compared with gold standard test (microscopy blood slide). Clinicians adhered to malaria guidelines by prescribing antimalarial drugs to patients with positive mRDT and did not adhere in case of negative mRDT result. The negative mRDT patients were prescribed with either antimalarial or antibiotics, while WHO 2010 guidelines recommend further investigation.

\section{REFERENCES}

[1] Roll Back Malaria Research Group. (2010). International Federation of Red Cross and Red Crescent Societies. Optimizing control of infectious diseases in resource-poor countries: Malaria diagnosis, fever home-based management and new tools. Brussels, Belgium. Available at: http://www.rbm.who.int/globaladvocacy.

[2] WHO. (2013). Global malaria programme. World malaria report 2013. Geneva. Available at: http:// www.who.int/malaria

[3] Derua, Y. A., Ishengoma, D. R. and Rwegoshora, R. (2011). Users' and health service providers' perception on quality of laboratory malaria diagnosis in Tanzania. Malaria Journal,
10:78. Available at: http://www.malariajournal.com/content/ $10 / 78$

[4] Ansah, E. K., Joanna, R., Samson, A., Christopher, J. M. and Chandler, C. I. R. (2013). Even if the test result is negative, they should be able to tell us what is wrong with us: A qualitative study of patient expectations of rapid diagnostic tests for malaria." Malaria Journal 12: 258. Available at: http://www.malariajournal.com/content/12/1/258

[5] Kyabayinze, D. J., Asiimwe, C., Nakanjako, D., Nabakooza, J., Counihan, H. and James, K. T. (2010). Use of RDTs to improve malaria diagnosis and fever case management at primary health care facilities in Uganda. Malaria Journal, 9: 200. Available at: http: //www.malariajournal.com/content/9 $/ 1 / 200$.

[6] Abeku, T. A., Kristan, M., Jones, C., Beard, J., Mueller, D. H., Okia, M., Rapuoda, B., Greenwood, B. and Cox, J. (2008). Determinants of the accuracy of rapid diagnostic tests in malaria case-management: Evidence from low and moderate transmission settings in the East African highlands. Malaria Journal, 7: 202. Available at: http://www.malariajournal.co $\mathrm{m} /$ content $/ 7 / 1 / 202$

[7] Davidson, H., Ndhlovu, M., Zurovac, D., Fox, M., Yeboah, A. K., Chanda, P., Sipilinyambe, N., Simon, J. L. and Snow, R. W. (2007). Improved diagnostic testing and malaria treatment practices in Zambia. The Journal of the American Medical Association, 297: 2227-2231. Available at: http://www.ncbi. nlm.nih.gov/pmc/articles/PMC2674546

[8] NMCP (2012). Training on malaria rapid diagnostic test to health care workers. Malaria control series 24. Ministry of Health and Social Welfare, United republic of Tanzania, 2012.

[9] Mangham, L. J., Cundill, B., Achonduh, O., Ambebila, J. N., Lele, A. K., Metoh, T. N., Ndive, S. N., Ndong, I. C., Nguela, R. L. and Nji, A. M. (2012). Malaria prevalence and treatment of febrile patients at health facilities and medicine retailers in Cameroon. Tropical Medicine \& International Health, 17: 330-42. Available at: http://www.ncbi.nlm.nih.gov/pubmed/ 22098135

[10] Ishengoma, D. S., Filbert, F., Mmbando, B. P., Lusingu, J. P. A., Magistrado, P., Michael, A., Theander, T. G. and Lemnge, M. M. (2011). "Accuracy of malaria rapid diagnostic tests in community studies and their impact on treatment of malaria in an area with declining malaria burden in North-Eastern Tanzania." Malaria Journal, 10: 176. Available at: http://www.malariajournal.com/content/10/1/176.

[11] Aydin, S. B., Mubi, M., Morris, U., Max, P., Ngasala, B. E., Premji, Z., Anders, B. and Andreas, M. (2013). Usefulness of Plasmodium falciparum specific rapid diagnostic tests for assessment of parasite clearance and detection of recurrent infections after artemisinin-based combination therapy. Malaria Journal, 12: 349. Available at: http://www.malariaj ournal.com/content/12/1/349

[12] Ngasala, B., Mubi, M., Warsame, M., Max, G. P., Massele, A.Y., Gustafsson, L. L., Goran, T., Premji, Z. and Anders, B. (2008). Impact of training in clinical and microscopy diagnosis of childhood malaria on antimalarial drug prescription and health outcome at primary health care level in Tanzania: A randomized controlled trial. Malaria Journal, 7: 199. Available at:http://www.malariajournal.com/content/ $7 / 1 / 199$ 
[13] Ifakara health institute. (2012). Evaluation of two malaria rapid diagnostic tests quality assurance (mRDT's) methods in peripheral health facilities, rural Tanzania. Available at: http://www. ihi.org.tz

[14] Huynh, B. T., Nadine, F., Gildas, G., Borgella, S., Blaise, G. M., Achille, M., Philippe, D. and Michel, C. (2011). Malaria associated symptoms in pregnant women followed-up in Benin. Malaria Journal, 10: 72. Available at: http://www.malariajournal.com/content/10/1/72

[15] Gosling, R. D., Christopher, J., Mwita, D. A. and Chandramohan, D. ( 2008). "Presumptive treatment of fever cases as malaria: Help or hindrance for malaria control?" Malaria Journal 7: 132 Available from: http://www.malariaj ournal.com/content/7/1/132

[16] Mutabingwa, T. K. (2005). Artemisinin-based combination therapies (ACTs): Best hope for malaria treatment but inaccessible to the needy. Malaria Journal, 95: 305-15. Available at: http://www.ncbi.nlm.nih.gov/pubmed/1609894 6

[17] Whitty, C., Hopkins, H. and Ansah, E. (2008). Opportunities and threats in targeting antimalarials: The role of diagnostics. The Affordable Medicines Facility. Washington. Available at: http://www.rff.org/RFF/Documents/RFF-DP-08-41.pdf
[18] Mubi, M., Kakoko, D., Ngasala, B., Premji, Z., Stefan, P., Anders, B. and Mårtensson, A. (2013). "Malaria diagnosis and treatment practices following introduction of rapid diagnostic tests in Kibaha district, Coast region, Tanzania." Malaria Journal, 12:293. Available at: http://www.malariajo urnal.com/content/12/1/293

[19] Batwala, V., Pascal, M. and Nuwaha, F. (2011). Comparative feasibility of implementing rapid diagnostic test and microscopy for parasitological diagnosis of malaria in Uganda. Malaria Journal, 10: 373. Available at: http://www.malariajournal.com/content/10/1/373

[20] Chandler, C. I. R., Mangham, L., Abanda, N., Olivia, A., Wilfred, F. M. and Wiseman, V. (2012). As a clinician, you are not managing lab results, you are managing the patient: How the enactment of malaria at health facilities in Cameroon compares with new WHO guidelines for the use of malaria tests. Social Science and Medicine, 74:1528-1535. Available at: http://www.sciencedirect.com/content/74/10/1528-1535.

[21] Acremont, V. D., Kilowoko, M., Kyungu, E., Philipina, S., Willy, S., Kahama, M. J., Lengeler, C., Pascal, C., Laurent, K. and Blaiser, G. (2014). Cause of fever in outpatient Tanzania children. Beyond malaria, 370: 09. Available at: http://www. Nejm.org/doi/full/10.1056/NEJMoa1214482. 